\title{
Physio- und Ergotherapie bei distalen Radiusfrakturen
}

\author{
Ingo Flesch, Cornelia Pfister, Kuno Weise
}

\section{Zusammenfassung}

Sowohl Physiotherapie als auch Ergotherapie stellen integrale Bestandteile der Behandlung nach distaler Radiusfraktur dar, um eine rasche Rückkehr des Patienten in seinen Alltag zu ermöglichen. Der Patient muss in seiner Selbstaktivität unterstützt werden, damit er wieder Vertrauen in seine verletzte Extremität fasst. Das Therapiekonzept muss möglichst viel Raum für Eigeninitiative des Patienten bieten.

\section{Einleitung}

Ziel physio- als auch ergotherapeutischer Behandlung ist die Wiederherstellung bestmöglicher Funktionen des verletzten Körperteils, um dem Patienten möglichst schnell die Rückkehr in den Alltag zu ermöglichen. Dies kann nur durch ein Therapiekonzept erreicht werden, das eine enge Kooperation zwischen dem Chirurgen und Therapeuten vorsieht.

Ein Teil dieser Zusammenarbeit beinhaltet den stetigen Informationsaustausch, um das Optimum für den Patienten zu erreichen. So wie Physio- und Ergotherapeuten über die Art und Stabilität einer Osteosynthese im Bild sein müssen, muss der verschreibende Chirurg über den Fortschritt, aber auch über die Stagnation der rezeptierten Behandlungen orientiert sein.

Der Kontakt des Physio- und Ergotherapeuten zu dem betroffenen Patienten

OP-JOURNAL 2003; 19: 66-69

(c) Georg Thieme Verlag Stuttgart · New York ist enger, weil zeitintensiver. Im Rahmen dieser Therapie besteht daher die Chance, dass der Patient wieder das notwendige Vertrauen in seine verletzte Extremität fasst. Dieses Vertrauen ist auch deshalb notwendig, da Physio- und Ergotherapie als therapeutische Hilfestellung verstanden werden können, um den Patienten in seiner Selbstinitiative professionell zu unterstützen.

\section{Aspekte der funktionellen Anatomie des Handgelenks}

Das Handgelenk muss als funktionelle Einheit zwischen den beiden Unterarmknochen, der proximalen und der distalen Handwurzelreihe gesehen werden. Erst die Artikulationen untereinander im Zusammenspiel mit einer komplexen ligamentären Verspannung ermöglichen die Beweglichkeit im Handgelenk. Die knöcherne Anatomie steht in enger Nachbarschaft zu Nerven, Gefäßen und Sehnen, so dass sich Störungen auf das Gesamt-System auswirken können.

Dorsalextension im Handgelenk ist die Summe aus überwiegender Dorsalextension (2/3) der radiocarpalen Gelenkfläche und $1 / 3$ der proximalen Gelenkfläche. Bei der Palmarflexion ist es invers.

Bei eingeschränkter Dorsalextension ist häufig auch eine Einschränkung der Radialabduktion festzustellen.

Bei Abduktionsbewegungen im Handgelenk gleitet die Handwurzelreihe in entgegengesetzter Bewegung zur Handbewegung. Das Scaphoid wird bei Radialabduktion nach volar, bei Ulnaabduktion nach dorsal verschoben, Pronationsund Supinationsbewegungen erfolgen über das proximale und distale Radioulnargelenk.

Gerade die Kenntnis dieser speziellen Gelenkanatomie unterstreicht die Notwendigkeit der Remobilisierung der Handwurzel nach distaler Radiusfraktur.

\section{Physiotherapie in der Frühphase}

Die Dystrophieprophylaxe ist von entscheidender Bedeutung. Im Vordergrund der therapeutischen Bemühungen stehen Schmerzlinderung und Entstauung bei postoperativem oder posttraumatischem Ödem. Dazu setzt die Physiotherapie verschiedene Maßnahmen ein. Die Anleitung des Patienten sollte praktisch durchgeführt werden. Die Lagerung spielt eine wichtige Rolle bei der Resorptionsverbesserung. Der Patient muss darauf achten, dass die Hand über dem Ellenbogen, der Ellenbogen über dem Schultergelenk gehalten wird. Es entsteht ein Gefälle, so dass Schwellung leichter resorbiert werden kann (z.B. Hochlagern mit Ablegen des Unterarmgewichtes auf dem Kopf). Unterstützen kann der Patient die Entstauung mit der aktiven Muskelpumpe. Beim Fingerspreizen und Fingerschließen wird die Handbinnenmuskulatur kontrahiert, beim Handschließen und Handöffnen wird die Muskelpumpe am Unterarm aktiviert. Die Blutzirkulation wird durch die Muskelaktivität erhöht und die Resorption der Schwellung unterstützt (Abb.1).

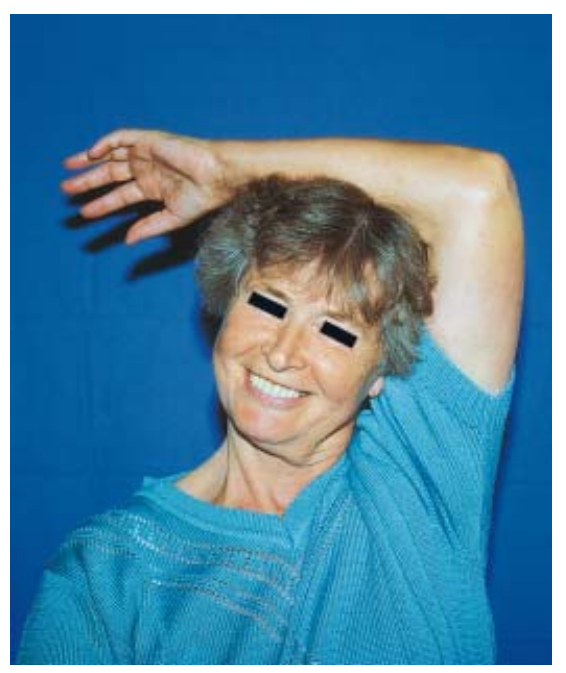

Abb.1 Resorptionsförderung durch Lagerung. 
Schmerzlinderung und Entstauung erreicht man auch durch Kälteanwendungen. Bei der Kurzzeiteisanwendung fängt man proximal der Verletzung (Schulter und Oberarm) an, mit einem Eisbeutel tupfend zu kühlen und arbeitet sich nach distal (Unterarm und Hand) vor. Der Patient muss adäquat angeleitet werden, er darf nur kühlen, nicht eisen.

Ist die Haut abgekühlt folgt eine Kühlphase, um die reaktive Mehrdurchblutung des Armes für den besseren und schnelleren Abtransport der Schwellungen auszunutzen. Mit zu starker Kälte wird oft der konträre Effekt einer Vasokonstriktion erreicht.

Kombiniert mit der Muskelpumpe verstärkt sich der Abtransport der Schwellung. Ist die Haut wieder erwärmt, wiederholt man den Kühlvorgang.

Die Fraktur ist durch Gips oder Osteosynthese ruhiggestellt. Dies erlaubt in der Physiotherapie den aktiven Erhalt der freien Beweglichkeit der an das Handgelenk angrenzenden Gelenke (Ellenbogen, Radioulnargelenke, Daumensattelgelenk, Fingergrundgelenke). Dabei darf die Gips- bzw. Schienenversorgung die endgradige Bewegung nicht einschränken. Besonderes Augenmerk muss auf die Umwendfunktion des Unterarms gelegt werden.

Dem Patienten sollte erklärt und gezeigt werden, dass er den verletzten Arm im erlaubten Ausmaß benutzen darf und muss. Dadurch wird eine übervorsichtige Schonung des verletzten Armes verhindert. Der aktive Einsatz z. B. beim Essen, bei der Körperpflege usw. verbessert die Trophik, und Arm und Hand bleiben oder werden im physiologischen Bewegungsverhalten integriert. Der Patient kann so wieder Vertrauen in seine verletzte Extremität fassen.

Sobald Bewegungsstabilität erreicht, wird das Handgelenk in die Physiotherapie einbezogen. Eine schonende Aktivierung ist das Bewegen von proximal, z.B. für die Dorsalextension im Handgelenk über den proximalen Hebel Unterarm. Über aktives und assistives Bewegen wird eine dosierte aktive Bewegungserweiterung im Handgelenk erreicht.

Für einen koordinierten Fingereinsatz ist eine aktive Widerlagerung im Handgelenk nötig. Das bedeutet, dass das Handgelenk in Dorsalextension aktiv muskulär stabilisiert wird, um eine physiologische, kombinierte Fingerflexion zu schulen.

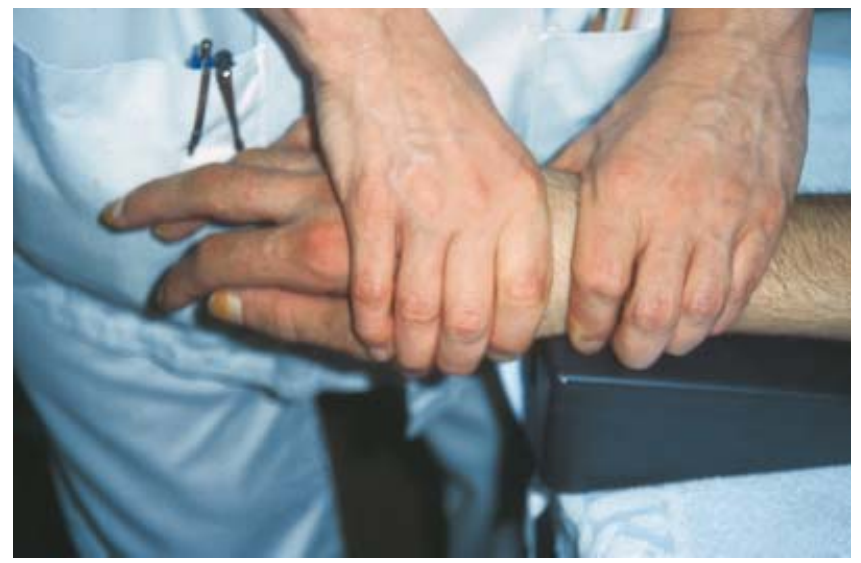

Abb. 2 Mobilisation der Handwurzel nach palmar für die Dorsalextension.

\section{Physio- und Ergotherapie in der Spätphase}

Die Fraktur ist belastungsstabil ausgeheilt.

Bei einem noch in der Bewegung eingeschränktem Handgelenk wird mit Manueller Therapie [1] durch Traktion und Mobilisation die Rollgleitbewegung im Gelenk und somit die Beweglichkeit verbessert (Abb. 2).

Nach dieser passiven Maßnahme muss das neu gewonnene Bewegungsausmaß sofort durch eine anschließende aktive Behandlungstechnik stabilisiert werden.

Man erarbeitet z. B. mit Propriozeptiver Neuromuskulärer Fazilitation (PNF) [2], eine Behandlungstechnik auf neurophysiologischer Basis, das Armmuster Extension/Adduktion/Innenrotation mit Ellenbogenflexion. Über Haltewiderstände auf dem Bewegungsweg und am Bewegungsende sowie über zeitliche und räumliche Summation werden die Beweglichkeit, Koordination und Kraft im ganzen Arm verbessert (Abb. 3).

Die Intensität wird durch den Einsatz von unterschiedlichen Muskelarbeitsweisen gesteigert: konzentrische, isometrische und exzentrische Muskelarbeit.

Die Stützaktivität des Armes wird erarbeitet durch steigerndes Belastungstraining. Es wird (z. B. mit PNF) im bilateralen Unterarmstütz gearbeitet, um den betroffenen Arm zu belasten. Im Seitstütz kommt noch mehr Belastung auf den Unterarm und das kann in Richtung Handstütz gesteigert werden (Abb.4).

Es werden Aktivitäten des täglichen Lebens, wie das Zusammenlegen des Behandlungslakens, in die Physiotherapie mit einbezogen. Die erlernten Bewegungsmöglichkeiten müssen benutzt, ausgenutzt und wieder in den Alltag integriert werden. Handgriffe und Bewegungsübergänge werden geschult z.B. über Schürze und Schuhe binden. Auch berufsspezifische Trainingmethoden werden in die Therapie integriert.

Zusätzlich zur physiotherapeutischen Einzelbehandlung wird im Medizinischen Aufbautraining [3] oder in der Krankengymnastik mit Gerät die betroffene Extremität mehr und mehr einbezogen. Der Patient wird zum Eigentraining angeleitet, um seine Kraftausdauer zu steigern, z.B. mit dem Theraband oder an den Trainingsgeräten wie Oberkörperergometer, Seilzugapparat, usw. (Abb. 5).

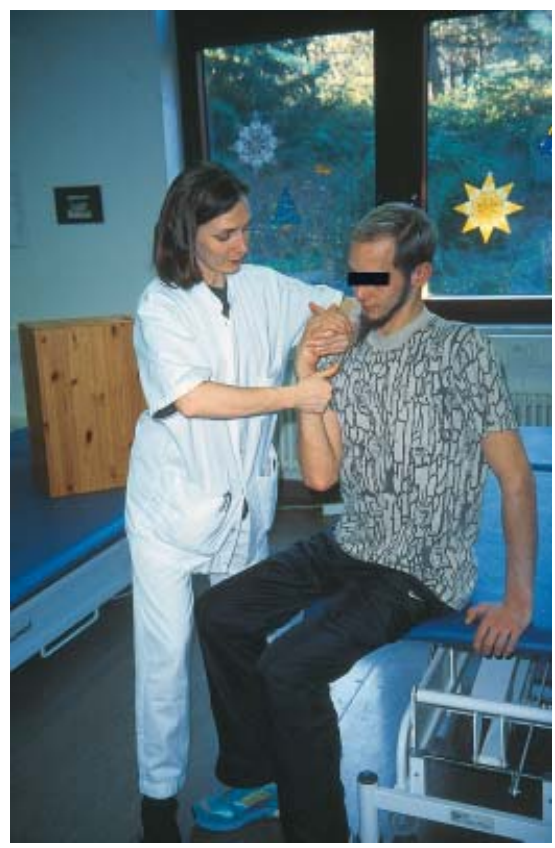

Abb.3 Propriozeptive neuromuskuläre Fazilitation. 


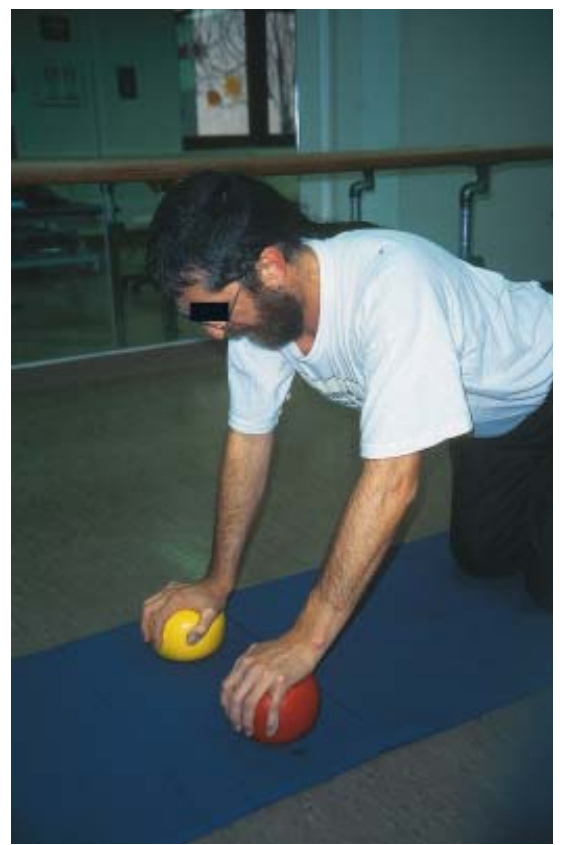

Abb.4 Handstütz im Vierfüßlerstand mit Ball wegen eingeschränkter Dorsalextension.

Sind die bisherigen Bemühungen ohne signifikanten Fortschritt geblieben oder hat sich das Vollbild einer Algodystrophie entwickelt, ergibt sich gelegentlich die Notwendigkeit, das Rehabilitationsprogramm unter stationären Bedingungen fortzusetzen.

Die manifeste Algodystrophie ist ein Zustand, der mit einem erheblichen Funktionsverlust der Hand einhergeht. Trotz intensiver Forschung auf dem Gebiet der Dystrophie ergeben sich bezüglich Ätiologie erhebliche Fragezeichen. Auch Hoffnungen medikamentöser Therapieformen (z.B. Calcitoningabe) haben sich nur zum Teil erfüllt. Neuere Forschungen

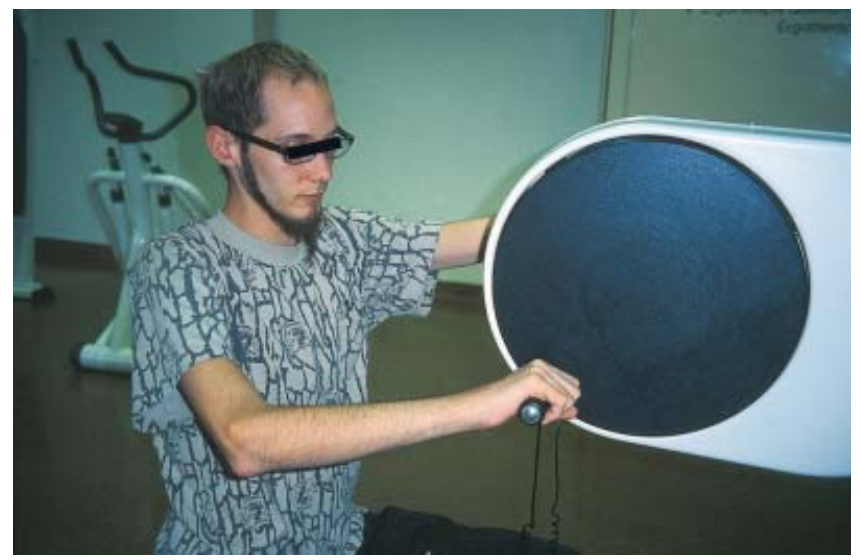

Abb. 5 Lokales Ausdauertraining am Oberkörperergometer. auf diesem Gebiet fokussieren auf das neurovaskuläre System. Der Schmerz wird nicht nur als Leitsymptom verstanden, sondern als mögliche Ursache des Symptomenkomplexes interpretiert.

Die Behandlung einer Algodystrophie sollte im Rahmen eines interdisziplinären Therapiekonzeptes erfolgen, in das die Schmerztherapie und die Rehabilitationsmedizin eingebunden ist. Nicht selten muss der Patient auch Hilfestellung von professioneller psychologischer Seite erhalten.

Befundabhängig wird dann der Therapieplan erstellt.

Im Rahmen des Heilverfahrens setzt man in der physiotherapeutischen Einzelbehandlung den Schwerpunkt je nach Befund und Ausmaß der Bewegungseinschränkungen in den Fingergelenken, Handgelenk, Radioulnargelenken, Ellenbogen oder Schulter in Bezug auf Mobilisation, Koordinationsschulung, Kräftigung und Handeinsatz.
Um die Trophik zu verbessern, erhält der Patient Wechselteilbäder für die obere Extremität. Auf die Trophik und den Schmerz kann man auch mit elektrotherapeutischen Maßnahmen einwirken. Bei starker Schwellung oder einem Ödem kommt Manuelle Lymphdrainage, evtl. mit einer Kompressionsbehandlung kombiniert, zum Einsatz. Schränkt eine rigide und adhärente Narbe die Bewegung ein, ist eine gezielte Narbenmassage hilfreich.

Hinzu kommt die Gruppenbehandlung im Bewegungsbad und in der Halle. Die Patienten erfahren den Vorteil der Gruppendynamik und erlernen so spielerisch den Handeinsatz. Ziel ist es, durch abwechslungsreiche Trainingseinheiten eine alltagsähnliche Belastung der Hand in Funktion und Ausdauer zu simulieren.

Das Gesamtbehandlungskonzept umfasst während beider Phasen zudem die Maßnahmen der Ergotherapie:

Neben funktionellen Übungen mit therapeutischer Knetmasse, Handgelenks-

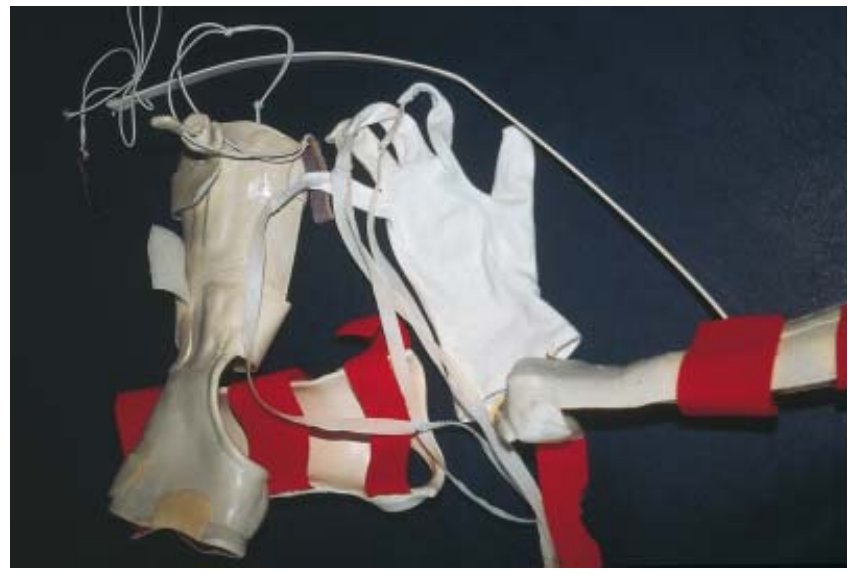

Abb.6a Beugehandschuh, Streck- und Beugequengel.

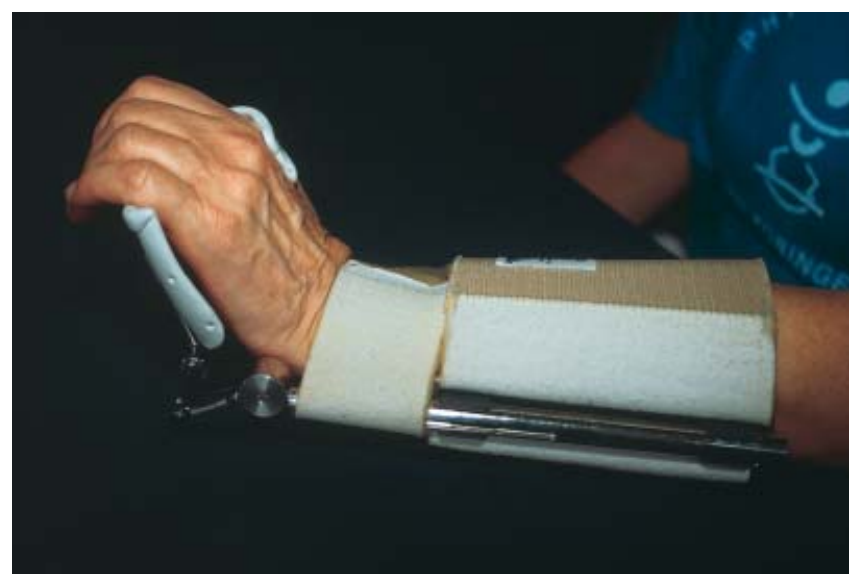

Abb.6b Quengelschiene für Dorsalextension. 
böckchen und anderen Medien lernt der Patient auch seine Hand wieder in alltagsähnlichen Situationen einzusetzen (Übungsküche, Holzwerkstatt). Auf berufsspezifische Tätigkeiten wird soweit wie möglich eingegangen.

Der Patient kann hierbei Koordination, Kraft, Gelenkbeweglichkeit und ein physiologisches Bewegungsverhalten wiedergewinnen.

Eine Schienenbehandlung kann die passive Bewegungserweiterung unterstützen. Schienen wie Beugehandschuh, Streck- und Beugequengel, sowie Schienen zur Verbesserung der Handgelenksbeweglichkeit werden individuell von den Ergotherapeuten angefertigt bzw. angepasst (Abb.6a, b).

\section{Schlussfolgerung}

Physiotherapie und Ergotherapie sind integrale Bestandteile des Therapieplans nach konservativ oder operativ behandelter Radiusfraktur, damit der Patient möglichst schnell die Funktion von Handgelenk und Hand wiedererreicht. Der stetige Informationsaustausch zwischen Arzt und Therapeuten ist hierbei essentiell.

\section{Literatur}

1 Bischoff HP. Manuelle Therapie für Physiotherapeuten. 3. Auflage, Demeter im Spitta Verlag Balingen 1998

2 Voss DE, Ionta MK, Myers BJ. Propriozeptive Neuromuskuläre Fazilitation, Bewegungsmuster und Techniken. 4. Auflage, Fischer, Stuttgart 1988

3 Radlinger L, Bachmann W, Homburg J, Lenenberger $U$, Thaddey G. Rehabilitatives Krafttraining. Thieme Stuttgart 1998
Dr. med. Ingo Flesch

Unfallchirurg

Cornelia Pfister

stellv. Abteilungsleitung

Physiotherapie

Prof. Dr. med. Kuno Weise

Ärztlicher Direktor

Berufsgenossenschaftliche Unfallklinik Schnarrenbergstraße 95

D-72076 Tübingen 